\title{
Effects of a reduction in the number of short intervals between work shifts on heart rate variability: A prospective field study of female nurses
}

\author{
Susanna Järvelin-Pasanen ${ }^{* 1}$, Tarja Hakola ${ }^{2}$, Harri Lindholm², Veikko Louhevaara ${ }^{1}$, Marja Paukkonen ${ }^{3}$, Sampsa \\ Puttonen $^{2,4}$, Annina Ropponen², Mika Tarvainen ${ }^{5,6}$, Tiina Pohjonen ${ }^{7}$ \\ ${ }^{1}$ University of Eastern Finland, Faculty of Health Sciences, School of Medicine, Institute of Public Health and Clinical Nutrition, \\ Ergonomics, Kuopio, Finland \\ ${ }^{2}$ Finnish Institute of Occupational Health, Helsinki, Finland \\ ${ }^{3}$ Health Centre, City of Helsinki, Helsinki, Finland \\ ${ }^{4}$ University of Helsinki, Institute of Behavioural Sciences, Helsinki, Finland \\ ${ }^{5}$ University of Eastern Finland, Faculty of Science and Forestry, Department of Applied Physics, Kuopio, Finland \\ ${ }^{6}$ Kuopio University Hospital, Department of Clinical Physiology and Nuclear Medicine, Kuopio, Finland \\ ${ }^{7}$ Helsinki City Occupational Health Centre, Helsinki, Finland
}

Received: April 1, 2015

DOI: $10.5430 /$ cns.v3n3p118
Accepted: May 22, 2015

Online Published: June 2, 2015

\begin{abstract}
Introduction: Shift work is one of the most serious occupational risk factors for health problems such as cardiovascular diseases. Furthermore, shift work disturbs sleep and alertness and impairs recovery from work, especially if the time between work shifts is insufficient. The aim of this study was to evaluate if a reduced number of short intervals (i.e., less than 11 hours) between work-shifts would result in better recovery indicated by sleep time heart rate variability reflecting the psychophysiological recovery.

Methods: Participants were 39 female shift-working nurses with the mean age of 45 years. The study design was a prospective within-subject study with a one year follow-up. The 24-hour heart rate variability recordings supplemented with questionnaires were performed twice. First, while working in the old shift schedule with frequent short intervals between work shifts, and again after one year of a working schedule with a reduced number of short intervals between work shifts. Statistical analyses were conducted using liner mixed models.

Results: The comparison between the initial shift system and the schedule with the reduced number of short intervals between work shifts caused an increase in heart rate variability parameters reflecting mainly parasympathetic activation of the autonomic nervous system (i.e., RMSSD, HF power and HF power in normalized units) $(p<.001)$.

Conclusions: In conclusion, our results suggest that reducing the number of short intervals between work shifts is an effective way to enhance the physiological recovery during the sleep. This study demonstrated that the recovery of autonomic nervous system from shift work can be promoted by implementation of ergonomic recommendations.
\end{abstract}

Key Words: Autonomic nervous system, Heart rate variability, Shift work, Scheduling

*Correspondence: Susanna Järvelin-Pasanen; Email: susanna.jarvelin@uef.fi; Address: University of Eastern Finland, Faculty of Health Sciences, School of Medicine, Institute of Public Health and Clinical Nutrition, Ergonomics, P.O. Box 1627, FI-70211 Kuopio, Finland. 


\section{INTRODUCTION}

In Europe, around one out of every five workers does shift work, at least occasionally. ${ }^{[1]}$ In particular, health care workers work in shifts and have irregular working hours. ${ }^{[1,2]}$ It is known that shift work is one of the most serious risk factors contributing to occupational health problems, ${ }^{[2,3]}$ for example being related to an increased risk of suffering cardiovascular diseases, ${ }^{[4-6]}$ breast cancer, ${ }^{[7]}$ mental problems, ${ }^{[8]}$ and occupational injuries. ${ }^{[9]}$ Furthermore, shift work disturbs sleep and alertness, ${ }^{[10,11]}$ and shift workers can experience difficulties in participating in family life as well as undertaking social, cultural, and other free-time activities. ${ }^{[8]}$ Moreover, shift work may disturb the recovery from work, especially if there is insufficient time for sleep and rest between the work shifts. ${ }^{[10,12]}$ An insufficient recovery can contribute to potential psycho-physiological and physical health complaints such as hypertension and cardiovascular diseases, ${ }^{[13-15]}$ as well as an increased risk for cardiovascular morbidity. ${ }^{[16]}$

Recovery is a process during which the psychophysiological systems revert to their baseline or initial level before the stressful situation. ${ }^{[14,17]}$ Insufficient recovery disturbs the normal balance of the autonomic nervous system (ANS) by increasing the sympathetic tone this being reflected in several physiological processes such as blood pressure and heart rate regulation. ${ }^{[13-15]}$ It is known that rest and recovery enhance the parasympathetic contribution to the ANS and that they increase heart rate variability (HRV). ${ }^{[18,19]}$

In shift work, recovery takes place after the daily working hours, e.g., during leisure-time and nighttime sleep, or during weekends and holidays. ${ }^{[17]}$ Shift systems with sufficient time for recovery seem to be favourable in terms of the quality of the sleep, and the subsequent reduced risk of fatigue, as well as causing less interference with social and family life. ${ }^{[10,20-22]}$ Furthermore, recovery from daily strain is determined by the work-rest cycle, ${ }^{[23]}$ and this can be influenced by several individual factors, such as lifestyle, socioeconomical and private life factors, stress coping skills ${ }^{[17]}$ and leisure time activities. ${ }^{[23,24]}$

HRV refers to the variability in the time intervals between successive normal-to-normal heart beats, i.e. RR time intervals. ${ }^{[18]} \mathrm{HRV}$ is calculated from long-term electrocardiographic recordings and it is a non-invasive method to assess cardiovascular autonomic regulation and changes related to psychophysiological strain and recovery. In addition, HRV analysis has been shown to be an effective method for assessing work-related stressors. ${ }^{[25]}$ In the analyses of HRV, many factors such as age, ${ }^{[26-28]}$ gender, ${ }^{[19,27]}$ cardiorespiratory fitness, ${ }^{[29]}$ health status, ${ }^{[19]}$ medication, ${ }^{[19]}$ and smoking ${ }^{[19]}$

Published by Sciedu Press need to be taken into account, because they all influence HRV. HRV has been widely used to assess psychophysiological recovery processes. ${ }^{[30,31]}$ The level of recovery after work can be evaluated by monitoring the changes in the HRV parameters, i.e. either an increase in parameters mainly affected by parasympathetic nervous activity or a decrease in the parameters mainly affected by sympathetic nervous activity (see Table 1). ${ }^{[25,30,32]}$ A study investigating workers with irregular shift work showed that a higher HRV during the first hours of sleep was associated with better subjective recovery. ${ }^{[30]}$ Hence, it has been claimed that these HRV parameters of recovery may be considered as useful in the identification and prevention of work-related stress. ${ }^{[30]}$ Moreover, it is possible that these HRV parameters can be utilized not only in measuring stress or recovery, but also in the evaluation and promotion of occupational health. However, little is known about HRV in real working life, and the associations between work-related factors and HRV have been predominantly based on cross-sectional studies. ${ }^{[33]}$ The previous investigations linking occupational health and HRV have commonly focused on the assessment of work-related factors, e.g. physical or chemical work environment and psychosocial workload, ${ }^{[33]}$ perceived stress or job strain due to shift work ${ }^{[25,34]}$ and not on the recovery. However, the analysis of sleep time HRV may reveal complementary information about the recovery process.

\section{Aim}

The aim of this study was to evaluate if a reduced number of short intervals (i.e., less than 11 hours) between work shifts would have any positive effects on sleep time HRV (i.e., sleep during the first four hours from the beginning of the main sleep period) reflecting the psychophysiological recovery by using a within-subject study design with a one year follow-up.

\section{MeTHODS}

\subsection{Design}

The subjects were nurses $(n=90)$ who worked in six acute or long-term care inpatient wards in municipal hospitals. Nurses worked on irregular two-shift (including morning shift from 7:00 a.m. to 2:30 p.m. and evening shift from 1:00 p.m. to 9:00 p.m.) or three-shift work (including morning, evening, and also a night shift from 9:00 p.m. to 7:30 a.m.) both with repeated quick returns (i.e., morning shifts immediately after evening shifts). The subjects voluntarily participated in the Healthy Working Hours Research and Development Project that has been described previously in detail elsewhere. ${ }^{[35,36]}$ In brief, the shift schedules were altered to meet the recommended ergonomic guidelines ${ }^{[12,37]}$ by reducing quick returns (i.e., fewer morning shifts following immediately 
after evening shifts and this influenced the number of short intervals (less than recommended 11 hours) between work shifts). ${ }^{[35,36]}$ The average interval between morning and evening shifts was 10 hours at baseline and follow-up. There was no change in the starting or ending times of work shifts, only the number of quick returns per schedule was reduced. The mean annual number of quick returns was as follows: 28 at baseline (range 9-49) and 15 in the follow-up (range $1-29$ ), i.e. the reduction was $55 \%$ (range $14-59 \%$ ).

Table 1. Description of the HRV parameters estimating parasympathetic activation and both sympathetic and parasympathetic activation ${ }^{[18]}$

\begin{tabular}{|c|c|}
\hline Parameters & Description \\
\hline \multicolumn{2}{|l|}{ HRV parameter (unit) } \\
\hline Mean RR interval (ms) & Mean of the selected beat-to-beat RR interval series, inversely proportional to mean heart rate \\
\hline \multicolumn{2}{|c|}{$\begin{array}{l}\text { Parameters estimating parasympathetic } \\
\text { activation }\end{array}$} \\
\hline RMSSD (ms) & $\begin{array}{l}\text { The square root of the mean of the squares of differences between consecutive RR intervals (describes } \\
\text { short-term variations) }\end{array}$ \\
\hline HF power(ms $\left.{ }^{2}\right)$ & High-frequency power (frequency range $0.15-0.4 \mathrm{~Hz}$ ) (synchronous with respiration) \\
\hline HF power (n.u.) & $\begin{array}{l}\text { High-frequency power in normalized units (n.u.) represent the relative power in proportion to the total power } \\
\text { minus the power of the very low frequency (VLF: } 0-0.04 \mathrm{~Hz} \text { ) component, HF power/(Total power-VLF power). }\end{array}$ \\
\hline \multicolumn{2}{|c|}{$\begin{array}{l}\text { Parameters estimating both sympathetic } \\
\text { and parasympathetic activation }\end{array}$} \\
\hline SDNN (ms) & $\begin{array}{l}\text { Standard deviation of all normal RR (normal-to-normal intervals, NN) intervals, the square root of variance } \\
\text { (demonstrates overall HRV) }\end{array}$ \\
\hline LF power (n.u.) & $\begin{array}{l}\text { Low-frequency power in normalized units (n.u.) represent the relative power in proportion to the total power } \\
\text { minus the power of the very low frequency (VLF: 0-0.04 Hz) component, LF power/(Total power-VLF power). }\end{array}$ \\
\hline $\mathrm{LF} / \mathrm{HF}$ & LF/HF ratio (estimates sympatho-vagal balance) \\
\hline
\end{tabular}

As a part of the study protocol, a medical evaluation was performed to define possible cardiovascular or other chronic diseases or medication with known effects on HRV (see Figure 1). The medical evaluation of the study subjects included a general health examination performed by an experienced physician and exercise test by bicycle ergometer conducted by a medical specialist in clinical physiology.

Subjects with missing $(n=38)$ or poor quality $(n=7) \mathrm{HRV}$ data were excluded from the analyses. The 45 excluded female subjects were similar to the subjects in final sample with respect to age and work experience. Because of the small number of male subjects $(n=6)$, they were also excluded from the final sample. Hence, the final sample consisted of 39 female nurses or vocational nurses for whom there was complete HRV data from both measurements (i.e., before and after the implementation of the new shift schedule with the reduced number of short intervals between work shifts) and without any cardiovascular or other chronic diseases or medication that could affect HRV (see Figure 1).

\subsection{Data collection}

The data were gathered through 24-hour HRV recordings with a diary and a questionnaire both before the implementation of the changes to the shift-work schedules (later referred to as the baseline) and about one year after the change (sub- sequently referred to as the follow-up).

\subsubsection{Heart rate variability recordings}

Suunto T6 (at baseline) and Suunto Smart Belt (at follow-up) heart rate (HR) monitors were used for recording the beatto-beat RR intervals. These HR monitors have been shown to be valid and reliable for recording HRV during physically and mentally stressful situations, and the reliability is known to be higher when used with chest electrodes. ${ }^{[38]}$

In the present study, the 24-hour HRV recordings were performed twice, at baseline while the nurses were working on the schedules with many short intervals and in the follow-up after the implementation of the schedules with reduced number of short intervals both during morning shift days. The time between the baseline and follow-up measurements was about one year.

The HRV recording periods started before the beginning of the work shift and ended about 24 hours later. Since, the HRV recordings were performed between successive work shifts they included the actual work shift (from 7:00 a.m. to 2:30 p.m.), leisure-time and the night after the work shift. In the analyses of HRV, the previous work shift that was available through the roster had to be taken into account, which were the morning shift ( $n=15$ and 19, at baseline and follow-up, respectively), evening shift ( $n=18$ and 4) or day 
off ( $n=3$ and 13). The information about the previous shift was not available in three cases at baseline and follow-up.

\subsubsection{Analyses of the HRV data}

HRV analysis related to recovery should optimally be conducted during slow wave sleep (SWS), ${ }^{[39]}$ which occurs mainly during the first half of the sleep period. ${ }^{[30]}$ Thus, the first four hours from the beginning of the sleep was selected for analysis. The beat-to-beat RR interval data of both the measurements were visually checked for artefacts such as missing beat detections or ectopic beats. Then artefactfree (i.e., artefact rate $<5 \%$ and correction for artifact beats using interpolation methods) 10 -min time periods during the first four hours from the beginning of the sleep were systematically selected for the analyses of each subject. HRV parameter values from the 10-min periods were finally averaged to obtain a mean HRV parameter for the four hour time period (see Figure 2).

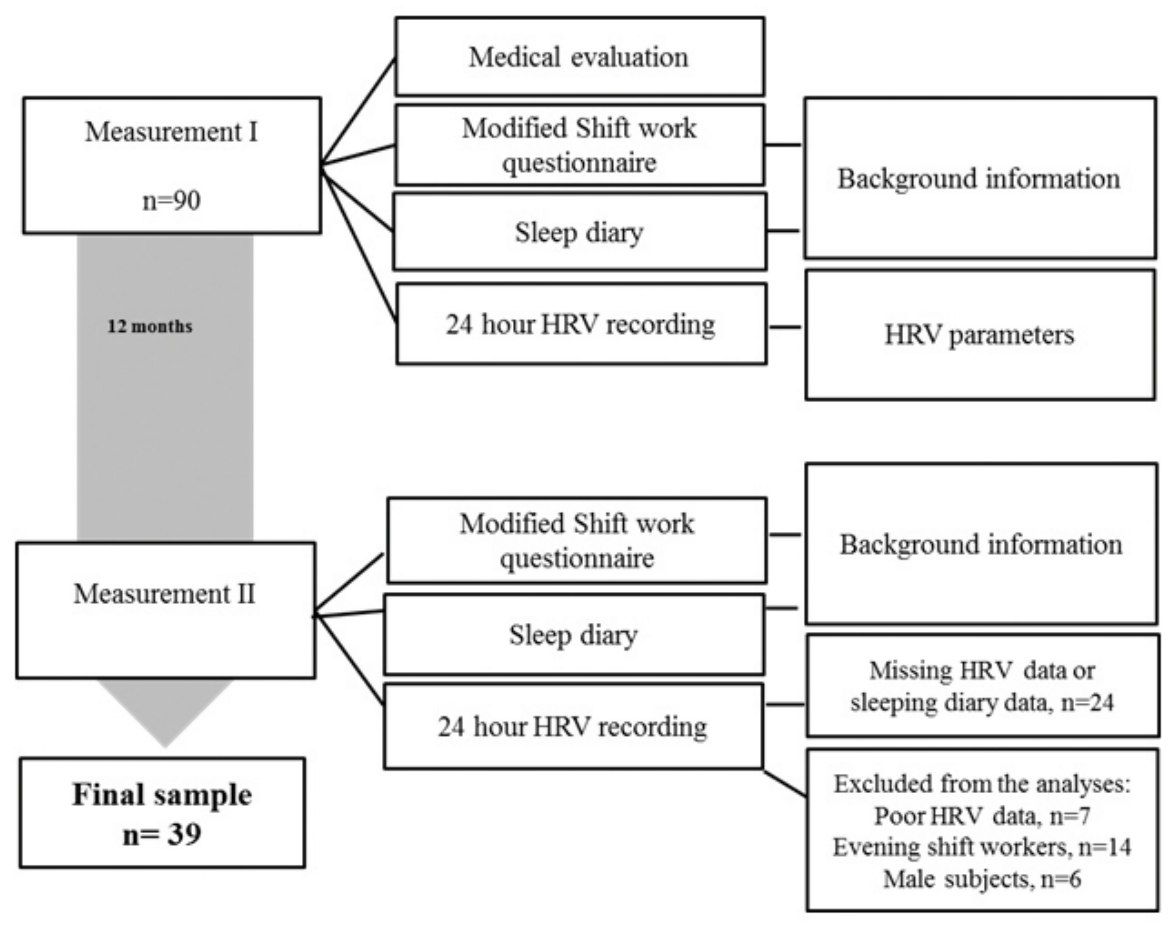

Figure 1. Study design

All HRV data were analyzed with Kubios HRV (ver. 2.1) software. ${ }^{[4]}$ Frequencies below $0.04 \mathrm{~Hz}$ were filtered out from the RR interval time series by using a smoothness priors detrending approach. ${ }^{[41]}$ Time-domain parameters such as the standard deviation of normal-to-normal RR intervals (SDNN) and root mean square of successive RR interval differences (RMSSD) were computed from the detrended time series. Before computations of frequency-domain HRV parameters, the beat-to-beat RR time series were interpolated using $4 \mathrm{~Hz}$ cubic spline interpolation in order to have equidistantly sampled data for spectral estimation. Spectrum estimates were then computed using Welch's periodogram method with a window width of 200 seconds and $50 \%$ overlap.

\subsubsection{Diary and questionnaire}

The subjects kept a diary during the 24-hour HRV recording period and were instructed to work in their habitual manner and to maintain their normal lifestyle. The information about the beginning and the end of the sleep and the quality of sleep were determined from the diary. The quality of sleep Published by Sciedu Press was rated on a 5 -point scale $1=$ very poor, $2=$ poor, $3=\bmod$ erate, $4=$ good and $5=$ very good (modified Basic Nordic Sleep Questionnaire). ${ }^{[42]}$ In addition, the subjects reported the extent of leisure-time physical activity, as well as the doses of coffee and alcohol in their diary.

In addition, the subjects filled in a questionnaire with information about age, weight, height, health status (i.e., number of diseases diagnosed by a physician), physical activity level, work experience and shift-work experience. Body mass index (BMI, $\mathrm{kg} / \mathrm{m}^{2}$ ) was computed from self-reported weight and height. Furthermore, the subjects' self-reported physical activity levels were scored in seven categories as follows: $1=$ sedentary, $2=$ minimally active, $3=$ middle active , $4=$ moderately active, 5 = vigorously active, $6=$ very vigorous and $7=$ competitive. Levels 4 and higher indicate that the individual was taking enough physical activity to confer health benefits. ${ }^{[43]}$ In the analyses, the categories of physical activity were dichotomized into "insufficient physical activity" (i.e. categories 1-3) and "sufficient physical activity" (i.e. categories 4-7). 


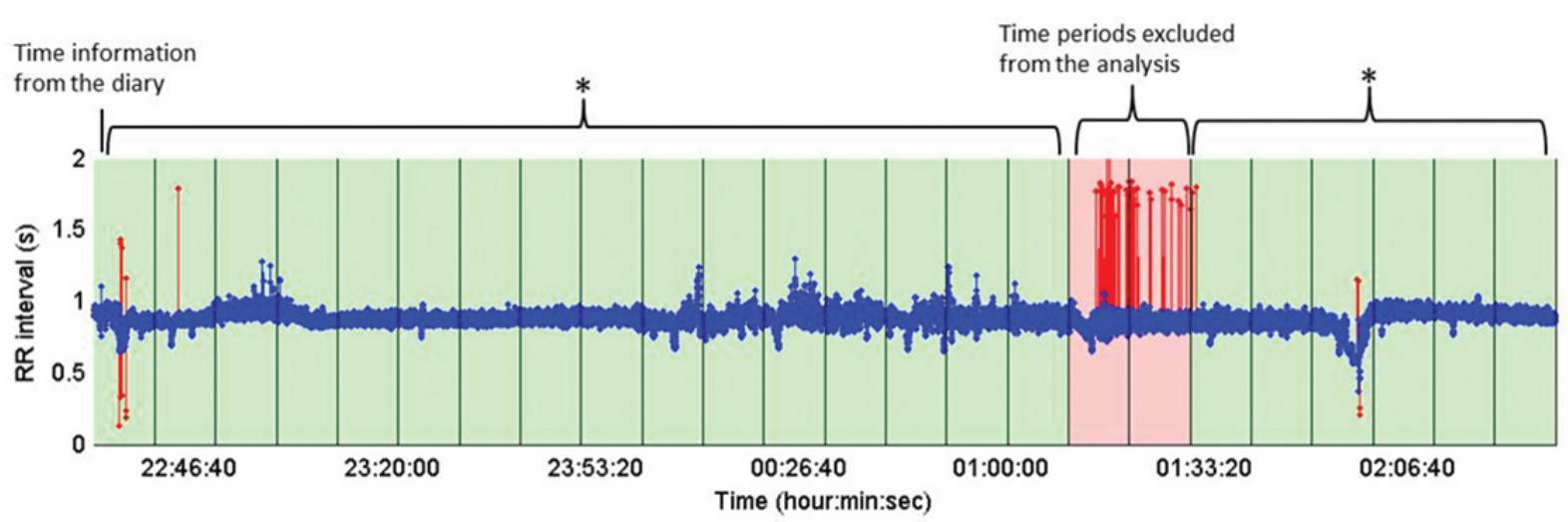

Figure 2. An example of HRV analysis

Artefact beat intervals (plotted in red) were corrected using interpolation methods. Time periods containing an excessive amount of artifacts (artefact rate $>5 \%$ ) were excluded from the analyses

\subsection{Statistical analyses}

Statistical analyses were performed using SPSS (version 19). Due to the skewed distributions, values of HRV were expressed as medians $(\mathrm{Md})$ and ranges. The HRV values were logarithmically transformed due to the skewness of the distribution. After the logarithmic transformation, the distribution of the data was verified by the Kolmogorov-Smirnov-test. Subsequently, the logarithmically transformed values were standardized to make the results more comparable (after standardization the mean of all parameters is zero and standard deviation is one). The linear mixed models were used to examine the associations between HRV parameters and the shift schedule type (the schedules with different number of short intervals between shifts). Each logarithmically transformed and standardized HRV parameter (meanRR, SDNN, RMSSD, LF power, LF power in normalized units, HF power, HF in normalized units and LF/HF) was used as a dependent variable and analyzed separately. In this prospective one year follow-up study, each subject served as her own control. Thus, the identification (ID) was used as a random effect in all analyses. In addition, age (in years) was used as a covariate in all analyses due to its known influence on HRV. ${ }^{[26-28]}$ Furthermore, physical activity (dichotomised as insufficient and sufficient physical activity) and the shift schedule type were used as factors in all analyses. In order to evaluate the potential effect of categorization, we tested the models with the original seven categories, but there was no marked effect on the association between self-reported leisure-time physical activity and sleep time HRV parameters (data not shown). Furthermore, we tested the effect of ward (i.e., acute or long-term care), the type of shift work (two-shift work or three-shift work), the work experience, experience in the shift work, the quality of sleep (1-5), the length of sleep, BMI $\left(\mathrm{kg} / \mathrm{m}^{2}\right)$, and lifestyle (i.e., smoking, doses of alcohol) as covariates in the models. Since there was no statistically significant influence of the ward, the type of shift work, the work experience, experience in the shift work, quality or length of sleep, BMI or lifestyle factors, they were excluded from the final analyses. In order to account for the potential confounding effect of the previous work shift, the interaction between the previous work shifts and the type of shift work was tested with a linear mixed model. The distribution of residuals was controlled in the analyses. The level of statistical significance was set to $p$-values less than .05 .

\subsection{Ethical considerations}

All the subjects signed a written informed consent form. The Ethics Committee of Helsinki University Hospital and the Research Committee of Health Centre, Helsinki (HUS 265/E0/05) approved the study protocol.

\section{Results}

At the baseline, the mean age of the subjects was 45 years (SD 9, range 23-59), and their average employment duration was 21 years (see Table 2). Most of the subjects had one physician-diagnosed disease (median 1, range 0-4).

Table 2. Means, standard deviations (SD) and ranges of the demographic characteristics, and work experience at the baseline $(n=39)$

\begin{tabular}{ll}
\hline Characteristic & Mean (SD, range) \\
\hline Age (years) & $45(9,23-59)$ \\
Body Mass Index $\left(\mathrm{kg} / \mathrm{m}^{2}\right)$ & $26.2(4.6,18.8-40.8)$ \\
Work experience (years) & $21(11,1-40)$ \\
Experience in shift work (years) & $18(9,1-33)$ \\
\hline
\end{tabular}

Descriptive data of the sleep time HRV parameters (median and ranges) for the baseline and follow-up are presented in Table 3. A significant increase between baseline and followup was detected in meanRR, SDNN, RMSSD, LF and HF 
power, and HF power in normalized units analyzed from the first four hours from the beginning of the sleep. Furthermore, a significant decrease between baseline and follow-up was detected in LF power in normalized units and LF/HF analyzed from the same time period (see Table 3 ).

There were no significant differences detected in any of the HRV parameters between the insufficient and sufficient physical activity groups (data not shown).

\section{Discussion}

The longitudinal sleep time HRV data of 39 female nurses was used to investigate the psychophysiological recovery related to number of short intervals between work shifts in one year follow-up. The main change attributable to the Healthy Working Hours Research and Development Project, was a reduced number of short intervals between work shifts. This was done in order to increase recovery times by excluding the short free times due to combinations of evening and morning shifts even though the number of total working hours (114 hours 45 minutes) and the amounts of weekend (one out of three free) and also evening and night work remained the same during each 3 -week schedule periods. ${ }^{[35]}$ The results of this study indicated that a reduced number of short intervals between work-shifts was associated with beneficial changes in HRV. In particular, the reduced number of short intervals resulted in increased sleep time RMSSD, HF power and HF power in normalized units. These results point to an increase in the parasympathetic with relation to sympathetic activation of the ANS occurring during the first four hours of sleep.

Table 3. Median (Md) and range for the HRV parameters and mean difference (95\% CI) in logarithmically transformed and standardized HRV values during the sleep (the mean of first four hours of the sleep) at baseline and follow-up

\begin{tabular}{lllll}
\hline Parameter & Baseline Md (range) & Follow-up Md (range) & Mean difference (95\% CI) & $\boldsymbol{p}^{\#}$ \\
\hline Mean RR (ms) & $719.3(509.9-860.5)$ & $921.9(745.3-1566.5)$ & $1.33(0.81,1.85)$ & .000 \\
SDNN (ms) & $27.9(7.8-77.9)$ & $45.4(14.8-105.5)$ & $1.49(0.89,2.08)$ & .000 \\
RMSSD (ms) & $16.9(4.7-126.5)$ & $39.6(12.0-95.5)$ & $1.45(1.00,1.91)$ & .000 \\
LF power (ms $\left.{ }^{2}\right)$ & $441.8(15.8-2786.4)$ & $949.0(113.8-6418.3)$ & $1.21(0.50,1.92)$ & .001 \\
LF power (n.u.) & $80.3(52.6-93.2)$ & $62.6(31.3-80.2)$ & $-1.14(-1.61,-0.67)$ & .000 \\
HF power (ms ${ }^{2 *}$ & $117.9(3.8-1291.1)$ & $547.0(42.9-3886.5)$ & $1.58(1.15,2.01)$ & .000 \\
HF power (n.u.) & $19.6(6.8-46.6)$ & $37.4(19.7-68.6)$ & $1.13(0.66,1.66)$ & .000 \\
LF/HF & $4.1(1.1-13.6)$ & $2.2(0.5-6.3)$ & $-0.67(-1.28,-0.06)$ & .033 \\
\hline
\end{tabular}

${ }^{*}$ Mean difference in logarithmically transformed and standardized HRV values between baseline - follow-up adjusted with age and physical activity, 95\%CI = 95\% Confidence Interval ${ }^{\#}$ Linear Mixed Model; ${ }^{* *}$ n.u. = normalized units LF / (Total power - VLF); ${ }^{* \#}$ n.u.= normalized units HF / (Total power - VLF)

Previous studies have indicated that the implementation of ergonomic recommendations for shift work can increase sleep length, and improve the quality of the sleep, perceived alertness, well-being at work and social and family life. ${ }^{[20,44]}$ In this study, the subjective sleep quality or sleep length were not related to sleep-time HRV (results not shown). The changes in the numbers of short intervals between workshifts increased the available time for recovery between successive work shifts and this is hypothesized to account for the positive effects on HRV at the beginning of the sleep, i.e., a decrease in the sympathovagal balance. The ergonomic working time arrangements have been shown to exert positive effects on the perceived physical, mental and social well-being of the nurses in all age groups based on the results of partially overlapping dataset as in this study. ${ }^{[35]}$ However, in the current study, we were able to observe the positive effects of ergonomic shift arrangements with objective measurements of sleep time HRV. In addition, our earlier results based on the partially same dataset indicated that the beginning of the work shift was less stressful and the psychophysiological strain of nurses was lower in the more ergonomic shift schedule as compared to the conventional shift schedule (i.e., increase in normalized HF power, and decrease in normalized LF and LF/HF ratio). ${ }^{[36]}$ An alternative explanation may be that the nurses had recovered better from the previous work shift when working in the ergonomic shift schedule.

In this study, there was a one year follow-up. Based on the previous knowledge, a one year follow-up is appropriate to detect the long-term changes. For example, according to the longitudinal study by van Amelsvoort et al. ${ }^{[31]}$ the changes in the autonomic nervous system function caused by shift work were seen after one year. However, in this prospective field study, the one year follow-up was selected for practical reasons. For example, it was wished to minimize the influence of seasonal variation or holidays.

As far as we are aware, the focus of earlier HRV studies of shift work has been on work time but the sleep time HRV 
has rarely been investigated. A recent report has indicated that the first hours of sleep could be useful in the assessment of recovery of ANS, ${ }^{[30]}$ because HRV analysis related to recovery is best done during the periods of SWS, which occurs mainly during the first half of the sleep period. ${ }^{[39]}$ Hence in this study, HRV was evaluated during the first four hours from the beginning of the sleep and this was anticipated to represent SWS of the subjects and, thus provide some stability in the measurements (i.e., during slow wave sleep, body movements or arousals are rare and respiratory patterns are regular). However, some concerns related to HRV cannot be resolved. So far, no generally accepted reference values of HRV parameters exist, despite the efforts to construct these references. ${ }^{[27]}$

The current technology for collecting HRV permits a reliable long-term (24-48 hours) data collection ${ }^{[38]}$ in real life circumstances such as during shift work, leisure-time and sleep. The HR monitors used in this study have been shown to have almost equal measurement accuracies as an ambulatory ECG system $^{[45]}$ although there are some claims that the ambulatory ECG system would be better in artefact identification and overall signal quality. Long term measurements with HR monitors are known to suffer from weak skin-electrode contact, and consequently, the measurements can sometimes include significant numbers of artifacts. This is a clear limitation of this measurement technology, often leading to decreased sample size. In this study, Suunto T6 (at baseline) and Suunto Smart Belt (at follow-up) HR) monitors were used for HRV recording. Their technical specifications are similar, but the Smart Belt has an internal memory on the chest belt, whereas the T6 saves the RR interval data on the wrist computer. This difference resulted in more missing beats due to transmission problems between the chest belt and the wrist computer in Suunto T6 compared to Suunto Smart Belt device. However, as the artefact periods were excluded from analysis this had no effect on the HRV results. The identification of R-R intervals is similar in both devices and the interbeat analysis was performed with same high-quality software.

This study had some limitations. Firstly, the sample size was relatively small and male subjects $(n=6)$ were excluded from the final sample. However, the size reduction in the final sample was very small, but limitation to only female nurses was done in order to improve homogeneity and to facilitate interpretations. This sample could be considered being healthy at baseline since nurses with cardiovascular and other diseases or medication with known effects on HRV were excluded from the study. Furthermore, the sample size of this study is similar to the HRV studies of shift work. ${ }^{[33]}$ In addition, the measurements were done under real life working conditions, 124 which meant that it was not possible to control environmental influences occurring during the measurement of HRV. For example, there may have been additional confounding due to factors such as timing of the previous work shift, quality of sleep during the night before the measurements, and stressful situations in life. In order to provide some control to the 24-hour recording periods, the subjects received instructions to maintain their habitual working manners and lifestyle, including possible smoking and caffeine intake, which are factors potentially influencing HRV. ${ }^{[19]}$ In addition, the subjects kept a diary during the HRV recording period. Based on the information in these diaries, three participants drank alcohol during HRV recordings (1-3 doses). Nine subjects were smokers and eleven subjects were taking regular medication; in the post hoc tests, their results did not differ from the others. However, the prospective study setting in which each subject served as her own control is likely to have minimized the impact of these factors.

When one wishes to quantify the state of the ANS, longterm HRV recordings offer many advantages. However, supplementary methods (e.g. subjective assessments and questionnaires) are important both in the interpretation of the HRV results and in controlling for the individual factors influencing HRV, such as the quality of sleep, ${ }^{[42]}$ age, ${ }^{[26-28]}$ cardiorespiratory fitness, ${ }^{[29]}$ health, ${ }^{[19]}$ medication, ${ }^{[19]}$ and smoking. ${ }^{[19]}$ The questionnaires of this study included both individual and work-related attributes, and hence they do provide relatively comprehensive coverage many of the factors potentially influencing the HRV parameters. This kind of data is needed since recovery of the ANS seems to be affected by several work- and leisure-related factors. ${ }^{[33]}$ Hence further studies with larger sample sizes will be needed to identify how HRV parameters can be influenced by either leisure-time or occupation related factors.

In conclusion, a reduction in the number of short intervals between work shifts resulted in significantly better recovery of female nurses as reflected by increased parasympathetic activity in relation to sympathetic HRV activity (i.e., RMSSD, $\mathrm{HF}$ power and HF power in normalized units) during the sleep. This study demonstrated that the recovery of autonomic nervous system from shift work can be promoted by implementation of ergonomic recommendations.

\section{ACKNOWLEDGEMENTS}

The Healthy Working Hours Research and Development Project was funded by the Finnish Work Environment Fund [project number 105273] and by the city of Helsinki (Health Centre and Human Resources Centre). S.J-P. was supported by the Finnish Work Environment Fund grants [project numbers 110005 and 114017].

ISSN 2324-7940 E-ISSN 2324-7959 


\section{REFERENCES}

[1] Parent-Thirion A, Vermeylen G, van Houten G, et al. Fifth European Working Conditions Survey. Publications Office of the European Union, Luxenbourg; 2012.

[2] Tucker P, Folkard S. Working Time, Health and Safety: A Research Synthesis Paper. Conditions of work and employment series No. 31. Geneva: Switzerland; 2012.

[3] Härmä M, Kecklund G. Shift work and health - how to proceed? Scand J Work Environ Health. 2010; 36: 81-84. PMID:20126970. http://dx.doi.org/10.5271/sjweh. 2902

[4] Knutsson A. Health disorders of shift workers. Occup Med (Oxford, England). 2003; 53: 103-108. http://dx.doi.org/10.1093/o ccmed/kqg048

[5] Puttonen S, Härmä M, Hublin C. Shift work and cardiovascular disease - pathways from circadian stress to morbidity. Scand J Work Environ Health. 2010; 36: 96-108. PMID:20087536. http: $/ / \mathrm{dx}$.doi.org/10.5271/sjweh.2894

[6] Vyas MV, Garg AX, Iansavichus AV, et al. Shift work and vascular events: systematic review and meta-analysis. BMJ (Clinical Research Ed.) 2012; 345: e4800. http://dx.doi .org/10.1136/bmj .e48 00

[7] Wang F, Yeung KL, Chan WC, et al. A meta-analysis on doseresponse relationship between night shift work and the risk of breast cancer. Ann Oncol. 2013; 24: 2724-2732. http://dx.doi.org/1 $0.1093 /$ annonc/mdt283

[8] Vogel M, Braungardt T, Meyer W, et al. The effects of shift work on physical and mental health. J Neural Transm. 2012; 119: 1121-1132. PMID:22488445. http://dx.doi.org/10.1007/s 00702-012-0800-4

[9] de Castro AB, Fujishiro K, Rue T, et al. Associations between work schedule characteristics and occupational injury and illness. Int Nurs Rev. 2010; 57: 188-194. http://dx.doi.org/10.1111/j.146 6-7657.2009.00793.x

[10] Härmä M. Workhours in relation to work stress, recovery and health. Scand J Work Environ Health. 2006; 2: 502-514. http: //dx.doi.org/10.5271/sjweh.1055

[11] Porkka-Heiskanen T, Zitting KM, Wigren HK. Sleep, its regulation and possible mechanisms of sleep disturbances. Acta Physiol (Oxf). 2013; 208: 311-328. http://dx.doi.org/10.1111/apha.1213 4

[12] Knauth P, Hornberger S. Preventive and compensatory measures for shift workers. Occup Med (Oxf). 2003; 53: 109-116. http: //dx.doi.org/10.1093/occmed/kqg049

[13] Lundberg U. Stress hormones in health and illness: the roles of work and gender. Psychoneuroendocrinology. 2005; 30: 10171021. PMID:15963652. http://dx.doi.org/10.1016/j . psyne uen.2005.03.014

[14] McEwen B. Protecting and damaging effects of stress mediators. $\mathrm{N}$ Eng1 J Med. 1998; 338: 171-179. PMID:9428819. http://dx.doi . org/10.1056/NEJM199801153380307

[15] Vrijkotte TG, van Doornen LJ, de Geus EJ. Effects of work stress on ambulatory blood pressure, heart rate, and heart rate variability. Hypertension. 2000; 35: 880-886. PMID:10775555. http: //dx.doi.org/10.1161/01.HYP.35.4.880

[16] Kivimäki M, Leino-Arjas P, Kaila-Kangas L, et al. Is incomplete recovery from work a risk marker of cardiovascular death? Prospective evidence from industrial employees. Psychosom Med. 2006; 68: 402-407. PMID:16738071. http://dx.doi.org/10.1097/0 1.psy.0000221285.50314.d3

[17] Geurts SA, Sonnentag S. Recovery as an explanatory mechanism in the relation between acute stress reactions and chronic health impairment. Scand J Work Environ Health. 2006; 2: 482-492. http://dx.doi.org/10.5271/sjweh.1053

[18] Task Force of the European Society of Cardiology and the North American Society of Pacing and Electrophysiology. Heart rate variability: standards of measurement, physiological interpretation and clinical use. Circulation. 1996; 93: 1043-1065. PMID:8598068. http://dx.doi.org/10.1161/01.CIR.93.5.1043

[19] Acharya UR, Joseph KP, Kannathal N, et al. Heart rate variability: a review. Med Biol Eng Comput. 2006; 44: 10311051. PMID:17111118. http://dx.doi.org/10.1007/s1151 7-006-0119-0

[20] Härmä M, Hakola T, Kandolin I, et al. A controlled intervention study on the effects of a very rapidly forward rotating shift system on sleep-wakefulness and well-being among young and elderly shift workers. Int J Psychophysiol. 2006; 59: 70-79. PMID:16297476. http://dx.doi.org/10.1016/j.ijpsycho.2005.08.005

[21] Sallinen M, Kecklund G. Shift work, sleep, and sleepiness - differences between shift schedules and systems. Scand J Work Environ Health. 2010; 36: 121-133. PMID:20119631. http://dx.doi.org $/ 10.5271 /$ sjweh. 2900

[22] Flo E, Pallesen S, Moen BE, et al. Short rest periods between work shifts predict sleep and health problems in nurses at 1-year follow-up. Occup Environ Med. 2014; 71: 555-561. http://dx.doi .org/10 1136/oemed-2013-102007

[23] Rook JW, Zijlstra FRH. The contribution of various types of activities to recovery. European Journal of Work and Organizational Psychology. 2006; 15: 218-240. http://dx.doi.org/10.1080/13594 320500513962

[24] Sonnentag S. Recovery, work engagement, and proactive behavior: a new look at the interface between nonwork and work. J Appl Psychol 2003; 88: 518-528. PMID:12814299. http://dx.doi.org/10.10 37/0021-9010.88.3.518

[25] van Amelsvoort LG, Schouten EG, Maan AC, et al. Occupational determinants of heart rate variability. Int Arch Occup Environ. 2000; 73: 255-262. http://dx.doi.org/10.1007/s004200050425

[26] Bonnemeier H, Richardt G, Potratz J, et al. Circadian profile of cardiac autonomic nervous modulation in healthy subjects: differing effects of aging and gender on heart rate variability. J Cardiovasc Electrophysiol. 2003; 4: 791-799. PMID:12890036. http: //dx.doi.org/10.1046/j.1540-8167.2003.03078.x

[27] Koskinen T, Kähönen M, Jula A, et al. Short-term heart rate variability in healthy young adults: the Cardiovascular Risk in Young Finns Study. Auton Neurosci. 2009; 145: 81-88. PMID:19019739. http://dx.doi.org/10.1016/j. autneu.2008.10.011

[28] Umetani K, Singer DH, McCraty R, et al. Twenty-four hour time domain heart rate variability and heart rate: relations to age and gender over nine decades. J Am Coll Cardiol. 1998; 31: 593-601. http://dx.doi.org/10.1016/S0735-1097 (97)00554-8

[29] Buchheit M, Gindre C. Cardiac parasympathetic regulation: respective associations with cardiorespiratory fitness and training load. Am J Physiol Heart Circ Physiol. 2006; 291: H451-458. PMID:16501030. http://dx.doi.org/10.1152/ajpheart.00008.2006

[30] Lindholm H, Sinisalo J, Ahlberg J, et al. Attenuation of vagal recovery during sleep and reduction of cortisol/melatonin ratio in late afternoon associate with prolonged daytime sleepiness among media workers with irregular shift work. Am J Ind Med. 2012; 55: 643-649. http://dx.doi.org/10.1002/ajim. 22042

[31] van Amelsvoort LG, Schouten EG, Maan AC, et al. Changes in frequency of premature complexes and heart rate variability related to shift work. Occup Environ Med. 2001; 58: 678-681. PMID:11555690. http://dx.doi.org/10.1136/oem.58.10.678 
[32] Wehrens SM, Hampton SM, Skene DJ. Heart rate variability and endothelial function after sleep deprivation and recovery sleep among male shift and non-shift workers. Scand J Work Environ Health. 2012; 38: 171-181. http://dx.doi.org/10.5271/sjweh.3197

[33] Togo F, Takahashi M. Heart rate variability in occupational health -a systematic review. Ind Health. 2009; 47: 589-602. PMID:19996534. http://dx.doi.org/10.2486/indhealth.47.589

[34] Langelotz C, Scharfenberg M, Haase O, et al. Stress and heart rate variability in surgeons during a 24-hour shift. Arch Surg. 2008; 143 751-755. http://dx.doi.org/10.1001/archsurg.143.8.751

[35] Hakola T, Paukkonen M, Pohjonen T. Less quick returns-greater well-being. Ind Health. 2010; 8: 390-394. http://dx.doi .org/1 $0.2486 /$ indhealth. MSSW-02

[36] Järvelin-Pasanen S, Ropponen A, Tarvainen M, et al. Effects of Implementing an Ergonomic Work Schedule on Heart Rate Variability in Shift-working Nurses. J Occup Health. 2014; 55: 225-233. http://dx.doi.org/10.1539/joh.12-0250-0A

[37] Knauth P. Changing schedules: Shiftwork. Chronobiol Int. 1997; 14: 159-171. PMID:9095376. http://dx.doi.org/10.3109/07420 529709001153

[38] Achten J, Jeukendrup AE. Heart rate monitoring: applications and limitations. Sports Med. 2003; 33: 517-538. PMID:12762827. http://dx.doi.org/10.2165/00007256-200333070-00004

[39] Brandenberger G, Buchheit M, Ehrhart J, et al. Is slow wave sleep an appropriate recording condition for heart rate variability anal- ysis? Auton Neurosci. 2005; 121(1-2): 81-86. PMID:16005265. http://dx.doi.org/10.1016/j.autneu.2005.06.002

[40] Tarvainen MP, Niskanen JP, Lipponen JA, et al. Kubios HRV - Heart rate variability analysis software. Comput Meth Prog Bio. 2014; 113: 210-220. http://dx.doi.org/10.1016/j.cmpb.2013.07.024

[41] Tarvainen MP, Ranta-Aho PO, Karjalainen PA. An advanced detrending method with application to HRV analysis. IEEE Trans Biomed Eng. 2002; 49: 172-175. PMID:12066885. http://dx.doi.org/1 $0.1109 / 10.979357$

[42] Partinen M, Gislason T. Basic Nordic Sleep Questionnaire (BNSQ): a quantitated measure of subjective sleep complaints. J Sleep Res. 1995; 4: 150-155. PMID:10607192. http://dx.doi.org/10.11 11/j.1365-2869.1995.tb00205.x

[43] Jackson AW, Morrow JJ, Hill DW, et al. Physical Activity for Health and Fitness. Updated Edition. Human Kinetics, Champaign: United States; 2004.

[44] Hakola T, Härmä M. Evaluation of a fast forward rotating shift schedule in the steel industry with a special focus on ageing and sleep. $\mathrm{J}$ Hum Ergol (Tokyo). 2001; 30: 315-319.

[45] Weippert M, Kumar M, Kreuzfeld S, et al. Comparison of three mobile devices for measuring R-R intervals and heart rate variability: Polar S810i, Suunto t6 and an ambulatory ECG system. Eur J App Physiol. 2010; 109: 779-786. http://dx.doi.org/10.1007/s00 421-010-1415-9 